J. Clin. Chem. Clin. Biochem.

Vol. 17, 1979, pp. 251-256

\title{
Gaschromatographische Bestimmung von Hydroxyprolin in biologischem Material des Menschen
}

Von W. Woiwode, Dagmar List und $H$. Weichardt

Aus dem Institut für Arbeits- und Sozialmedizin (Direktor: Prof. Dr. med. H. Weichardt) der Universität Tübingen

(Eingegangen am 28. August/15. November 1978)

Zusammenfassung: Die Methode der gaschromatographischen Aminosäureanalyse wurde auf die Bestimmung von $L$-Hydroxyprolin in biologischem Material des Menschen übertragen.

Die quantitative Bestimmung von $L$-Hydroxyprolin kann durch gaschromatographische Enantiomerentrennung nach Zusatz einer bekannten Menge allo- $D$-Hydroxyprolin zur Probe erfolgen, da dieser chemisch äquivalente Standard während der Derivatisierung allen Veränderungen in gleicher Weise wie die zu bestimmende $L$-Verbindung ausgesetzt ist. Die Vorteile gegenüber bisherigen Bestimmungsmethoden liegen in der höheren Genauigkeit, Reproduzierbarkeit und im geringeren Probenbedarf.

Gleichzeitig ist diese Arbeit ein Anwendungsbeispiel für chirale polymere Phasen, die es gestatten, die Aminosäurederivate einer Probe gaschromatographisch nicht nur untereinander, sondern auch in die jeweiligen Enantiomerenpaare aufzutrennen.

\section{Quantitative analysis of L-hydroxyproline in human biological material by gas-chromatography}

Summary: Quantitative amino acid analysis by gas chromatography was applied to the determination of 4-hydroxy$L$-proline in biological material.

After addition of a known amount of allo-4-hydroxy- $D$-proline, quantitative analysis of 4-hydroxy- $L$-proline can be performed by gas chromatographic separation of the amino acid enantiomers; since the standard and sample have identical chemical behaviour, they respond identically during derivatization. The accuracy, reproducibility, and sample requirement of this procedure are more advantageous than those of hitherto existing methods.

This study is also an example of the application of chiral stationary phases, which permit the simultaneous separation of amino acids and amino acid enantiomers.

\section{Einfuhihung}

Freie und gebundene Aminosäuren treten im Blut und im Urin in unterschiedlichen Konzentrationen auf. Während die quantitative Erfassung jeder einzelnen Aminosäure auf Grund der komplexen physiologischen Zuşammenhänge fuir diagnostische Belange (1) im allgemeinen noch nicht sinnvoll ist, kommt der Hydroxyprolinbestimmung als Parameter des Kollagenistoffwechsels (2) große Bedeutung zu. So ermöglicht etwa die Kenntnis der Hydroxyprolinkonzentration bzw. -menge im Blut und im Urin Aussagen über den Kollagenstoffwechsel unter physiologischen und pathologischen Bedingungen.

In der Vergangenheit wurden zahlreiche, auf photometrischen und gaschromatographischen Prinzipien ba- sierende Bestimmungsmethoden entwickelt, von denen hier nur einige angeführt sein sollen $(3,4,5)$.

Da diese Methoden z. T. aufwendig und relativ ungenau sind, übertrugen wir die von H. Frank, G. J. Nicholson $\& E$. Bayer entwickelte Methode der gaschromatographischen Aminosäureanalyse (6) auf die Hydroxyprolinbestimmung, um eine genaue und reproduzierbare Analyse bei vertretbarem Zeitaufwand durchführen zu können.

\section{Material und Methoden \\ Gaschromatographische Enantiomerentrennung (7)}

Die Verwendung von Glaskapillarsäulen, belegt mit der optisch aktiven Phase Chirasil-Val (8) gestattet es, durch Wahl eines geeigneten Temperaturprogrammes die Aminosäurederivate 
einer Probe gaschromatographisch nicht nur untereinander, sondern auch in die jewveiligen Enantiomerenpaare aufzutrennen.

Eine racemische Aminosäure ergibt somit, bei völlig gleichen chemischen Eigenschaften des $D$ - und $L$-Enantiomers, zwei Peaks im Chromatogramm.

$\mathrm{Da}$ in biologischem Material fast ausschließlich $L$-Aminosäuren gefunden werden, ist es möglich, durch Zusatz eines D-Aminosäuren-Testgemisches als Standard zur Probe jeder Aminosäure einen „eigenen" inneren Standard hinzuzufügen.

Erfolgt dieser Zusatz noch vor der Derivatisierung der Probe, kann die quantitative Bestimmung direkt aus dem Verhältnis der jeweiligen $D$ - und $L$-Peaks erfolgen, da der - chemisch gleiche - Standard unter den identischen Aufarbeitungsbedingungen allen Veränderungen in gleicher Weise unterworfen ist. Auch eine eventuelle Racemisierung der $\mathrm{zu}$ bestimmenden Aminosäure während der Derivatisierung $(9,10)$ kann über eine zweite gaschromatographische Enantiomerentrennung, durchgeführt ohne Zusatz des D-Aminosäuren-Testgemisches, korrigiert werden. Dieses Verfahren wurde im Folgenden auf die Bestimmung von Hydroxyprolin angewendet.

Vorbereitung der Urinproben

$5 \mathrm{ml}$ Urin werden mit $5 \mathrm{ml} 10 \mathrm{~mol} / \mathrm{l} \mathrm{HCl}$ in einer zugeschmolzenen Glasampulle 14 Stunden bei $110^{\circ} \mathrm{C}$ hydrolisiert.

Aliquote Anteile dieser Lösung werden für die gaschromatographische Bestimmung von freiem und gebundenem Hydroxyprolin direkt derivatisiert bzw. weiteren Reinigungsschritten unterworfen.

Reinigung der Proben

durch Ionenau stau sch chromatographie (11)

Vorbereitung des Kationenaustauschers DOWEX 50 WX 8 ( $H^{+}$-Form)

Das Austauscherharz wird mit $3 \mathrm{~mol} / 1 \mathrm{NH}_{4} \mathrm{OH}$ bedeckt, $30 \mathrm{~min}$ bei Raumtemperatur gerührt und auf einer Glasfritte D 3 scharf abgesaugt. Dieser Vorgang wird noch zweimal wiederholt.

Anschließend wird auf der Glasfritte mit bidest. Wasser bis zur neutralen Reaktion ausgewaschen.

Die Regeneration des Austauschers erfolgt mit $3 \mathrm{~mol} / 1 \mathrm{HCl}$. Dazu wird wie oben beschrieben dreimal je $30 \mathrm{~min} \mathrm{mit} 3 \mathrm{~mol} / \mathrm{l}$ $\mathrm{HCl}$ bei Raumtemperatur gerührt und abgesaugt. Mit bidest. Wasser wird neutral gewaschen.

\section{Vorbereitung des Anionenaustauschers DOWEX 1 X 8 (Acetat-Form)}

Das Austauscherharz wird wie oben beschrieben mit $3 \mathrm{~mol} / \mathrm{l}$ KOH dreimal je 30 min bei Raumtemperatur gerührt und abgesaugt.

Auf der Glasfritte wird annähernd neutral gewaschen.

Die Regeneration des Austauschers erfolgt mit $3 \mathrm{~mol} / \mathrm{l}$ $\mathrm{CH}_{3} \mathrm{COOH}$.

Dazu wird wie oben beschrieben dreimal je $30 \mathrm{~min}$ mit $3 \mathrm{~mol} / \mathrm{l}$ $\mathrm{CH}_{3} \mathrm{COOH}$ bei Raumtemperatur gerührt und abgesaugt.

Mit bidest. Wasser wird neutral gewaschen.

Mit den so vorbehandelten Austauschern werden Glassäulen mit den Abmessungen $1 \mathrm{~cm} \times 15 \mathrm{~cm}$ etwa zur Hälfte gefüllt.

Mit bidest. Wasser wird äquilibriert.

\section{Reinigung über den Kationenaustauscher}

$10 \mathrm{ml}$ Urinhydrolysat werden zur Trockene eingedampft, in

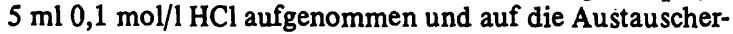
säule gegeben. Anschließend wird mit einer Geschwindigkeit von etwa $1 \mathrm{ml} / \mathrm{min}$ eluiert und noch dreimal mit je $2 \mathrm{ml}$ $0,1 \mathrm{~mol} / 1 \mathrm{HCl}$ nachgewaschen. Anschließend wird fünfmal mit je $10 \mathrm{ml}$ bidest. Wasser bei einer Geschwindigkeit von $5 \mathrm{ml} / \mathrm{min}$ eluiert. Das Eluat wird verworfen. Die Aminosäuren werden mit $3 \mathrm{~mol} / 1 \mathrm{NH}_{4} \mathrm{OH}$ eluiert. Dazu werden fünf separate Volumina von je $2 \mathrm{ml} \mathrm{NH}_{4} \mathrm{OH}$ auf die Säulen gegeben. Die
Elution erfolgt mit $3 \mathrm{ml} / \mathrm{min}$. Es wird noch fünfmal mit je $5 \mathrm{ml}$ bidest. Wasser nachgewaschen. Die die Aminosäuren enthaltenden alkalischen Fraktionen werden vereinigt.

Die Bestimmung von freiem und gebundenem Hydroxyprolin kann aus aliquoten Anteilen dieser Lösung erfolgen. Die verbliebene Lösung wird für die Trennung über den Anionenaustauscher zur Trockene eingedampft.

\section{Reinigung über den Anionenaustauscher}

Der eingedampfte Rückstand nach der Kationenaustauscher-

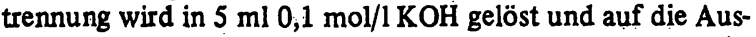
tauschersäule gegeben. Mit einer Geschwindigkeit von $2 \mathrm{ml} / \mathrm{min}$ wird eluiert.

Die Säule wird anschließend mit insgesamt $200 \mathrm{ml}$ bidest. Wasser bei einer Durchflußgeschwindigkeit von $5 \mathrm{ml} / \mathrm{min}$ gewaschen.

Die Aminosäuren werden mit $3 \mathrm{~mol} / 1 \mathrm{CH}_{3} \mathrm{COOH}$ eluiert. Dazu werden bei einer Geschwindigkeit von $3 \mathrm{ml} / \mathrm{min}$ zunächst fünfmal je $3 \mathrm{ml} \mathrm{CH}_{3} \mathrm{COOH}$ auf die Säule gegeben, anschließend noch fünfmal je $5 \mathrm{ml}$ bidest. Wasser.

Die die Aminosäuren enthaltenden Fraktionen werden vereinigt und eingedampitt.

Aliquote Anteile des Rückstandes werden für die gaschromatographische Enantiomerentrennung verwendet.

\section{Vorbereitung der Plasmaproben}

Zur Abtrennung von Proteinen wird $1 \mathrm{ml}$ Blutplasma mit $5 \mathrm{ml}$ wäßriger.Pikrinsäurelösung $(10 \mathrm{~g} / \mathrm{l})$ versetzt und fünf Minutèn bei Raumtemperatur gerührt. Die Suspension wird anschließend zehn Minuten bei $3500 \mathrm{U} / \mathrm{min}$ zentrifugiert. Die die Aminosäuren und überschüssige Pikrinsäure enthal tende überstehende klare Lösung wird dekantiert.

Die klare Lösung wird zur Abtrennung der Pikrinsäure wie oben beschrieben auf den Kationenaustauscher DOWEX 50 gegeben.

Derivatisierung der Proben

für die gaschromatographische Racemattrennung

Aliquote Anteile der jeweiligen Probenlösung werden eingedampft.

Ausgehend von $1 \mathrm{ml}$ Urin oder Plasma liegen die absoluten Stoffmengen der einzelnen Aminosäuren etwa zwischen 0,05 uṇd 0,5 Mikromol.

\section{Veresterung}

Der trockene Rückstand wird mit $0,5 \mathrm{ml}$ einer Lösung, die $1,5 \mathrm{~mol} \mathrm{HCl}$ in 11 Propanol- (2) enthält, versetzt und, sofern erforderlich, durch Ultraschallbehandlung homogenisiert. Die Probe wird auf $110^{\circ} \mathrm{C}$ erhitzt und 60 Minuten bei dieser Temperatur belassen.

\section{Acylierung}

Nach der Veresterung wird das Lösungsmittel mit Stickstoff abgeblasen. Nach Aufnehmen in $250 \mu 1$ Methylenchlorid und Zugabe von $50 \mu \mathrm{l}$ Perfluorpropionsäureanhydrid wird auf $110^{\circ} \mathrm{C}$ erhitzt und 10 Minuten bei $110^{\circ} \mathrm{C}$ belassen. Nach Abblasen bis zur Trockene mit Stickstoff wird die Probe zur Analyse in etwa $250 \mu \mathrm{l}$ Methylenchlorid aufgenommen.

\section{Gaschromatographische Racemattrennung}

Die Messungen wurden an einem Gaschromatographen der Firma Carlo Erba, Modell $2151 \mathrm{AC}$, ausgerisstet mit einem. Integrator der Firma Spectra-Physics, System 1, durchgefuhrt.

Ausgehend von 0,4 $\mu 1$ des Probengemisches erfolgen die Trennungen auf einer Glaskapillarsäule mit den Abmessungen $20 \mathrm{~m} \times 0,3 \mathrm{~mm}$, die nach bekannten Vorschriften (12) mit Chirasil-Val (13) belegt worden war. Chirasil-Val besteht aus einem Organosiloxan, an das ein L-Aminosäurederivat, in diesem Fall $L$-Val-tert.-butylamid, peptidartig geknüpft ist (8). Die Zahl der theoretischen Böden der benutzten Säule lag bei etwa 65000 . 

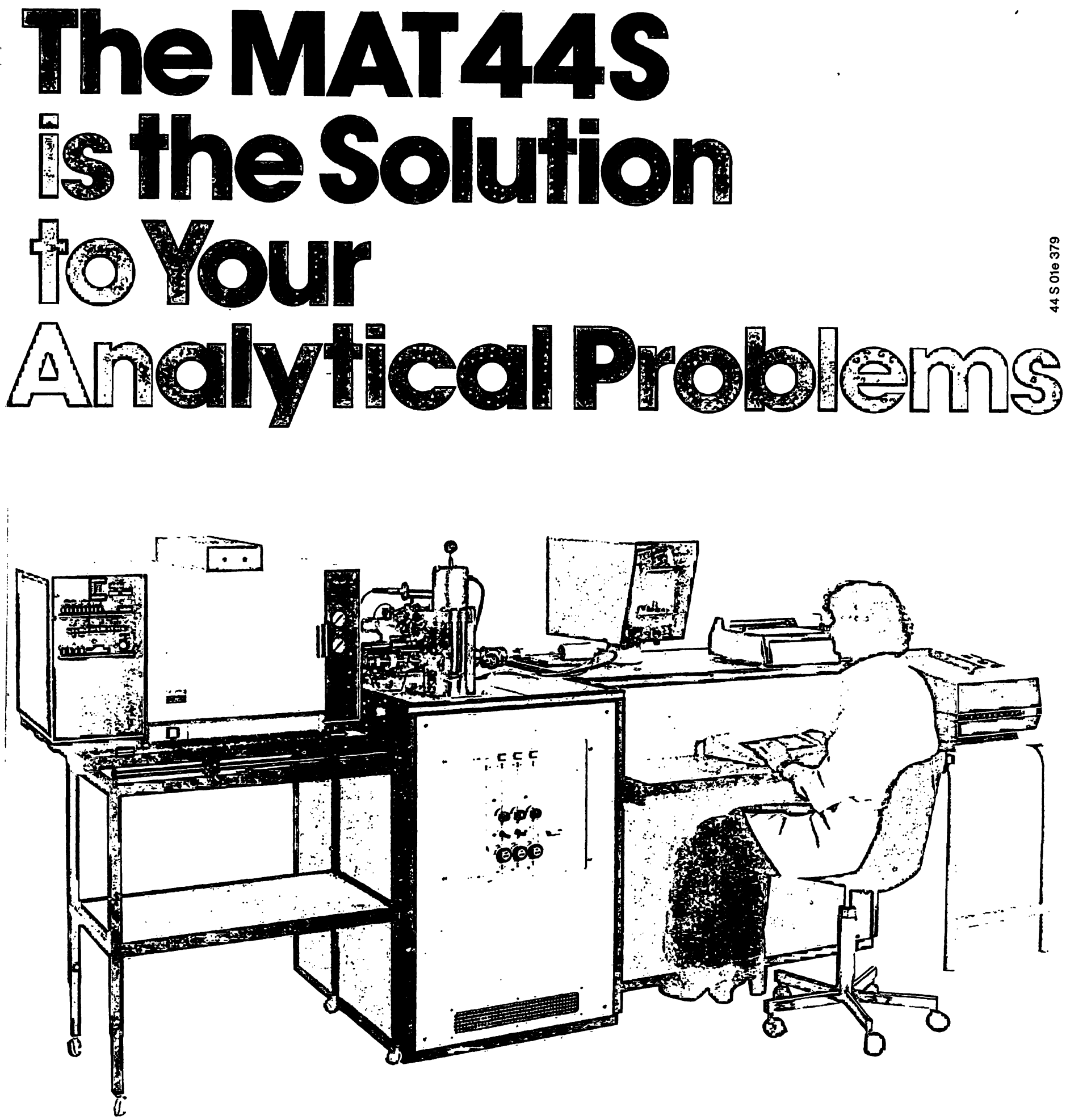

\section{CC/MS system MAT4AS}

*Our GC/MS/DS package prices will also ease your funding problems.

See the MAT44S at the ACHEMA'79 exhibition: hall 6/111, booth FG1-4. Frankfurt, June 17-23,79.
Varian MAT GmbH, Postfach 144062

Barkhausenstr.2,2800 Bremen 14

F.R.Germany, Telefon(O421)

VarianMATMass Spectrometry,

25 Hanover Rd., Florham Park, NJO7932.

Phone (201) 822-3700,U.S. 
- Autoklaven

HEINICKE INSTRUMENTS

Laborgerätebau $\mathrm{GmbH}$

8223 Trostberg, Pf. 1203

Ruf $08621 / 2085$, FS 563110

- Bakteriologie

GTTO NCRDLAALD KG

2000 Hamburg 50, Heinrichstr. 5

Ruf 040/4328 27

- Brutschränke

HEINICKE INSTRUMENTS

Laborgerätebau GmbH

8223 Trostberg, Pf. 1203

Ruf $08621 / 2085$, FS 563110

- Chromatographie-Pumpen VERDER (DEUTSCHLAND) GMBH 4000 Düsseldorf, Luegallee 108

Ruf $0211 / 574079$

FS: 08585539

- Digitale Verdünnungsgeräte

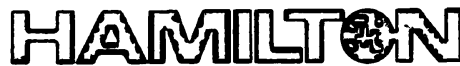

Hamilton Deutschland GmbH

Postfach 110427

Otto-Röhm-Str. 74

D-6100 Darmstadt

Ruf $06151 / 85085-86$, FS 0419684 .

- Digitale Dispensiergeräte

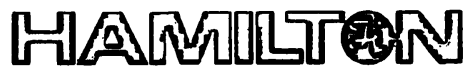

Hamilton Deutschland GmbH

Postfach 110427

Otto-Röhm-Str. 74

D-6100 Darmstadt

Ruf $06151 / 85085-86$, FS 0419684

- Elektrophorese

CARL ZEISS

7082 Oberkochen, Pf. 1369

- Filterphotometer

CARL ZEISS

7082 Oberkochen, Pf. 1369

- Flammenphotometer

CARL ZEISS

7082 Oberkochen, Pf. 1369

- Gasdichte Spritzen MAMLTEN

Hamilton Deutschland $\mathrm{GmbH}$

Postfach 110427

Otto-Röhm-Str. 74

D-6100 Darmstadt

Ruf 061 51/85085-86, FS 0419684
- Infrarot-Spektralphotometer CARL ZEISS

7082 Oberkochen, Pf. 1369

- Klinische Reagenzien

F PIERCE EUROCHEMIE B.y. P O BOX IISI-ROTTEROAM THE NETHERLANDS

- Küvetten

HELLMA GMBH \& CO.

7840 Mülheim/Baden

Ruf $07631 / 5509$ und 5500 FS 0772987

- Küvetten-Absaugpumpen

HELLMA GMBH \& CO.

7840 Müllheim/Baden

Ruf $07631 / 5509$ und 5500 FS 0772987

- Küvetten-Reinigungsmittel HELLMA GMBH \& CO.

7840 Müllheim/Baden

Ruf $07631 / 5509$ und 5500 FS 0772987

- Küvetten-Ständer

HEELMA GMBH \& CO.

7840 Müllheim/Baden

Ruf $07631 / 5509$ und 5500 FS 0772987

- Küvetten-Trockenschleudern

HELLMA GMBH \& CO.

7840 Müllheim/Baden

Ruf $07631 / 5509$ und 5500

FS 0772987

\section{- Laborspülmittel}

deconex ${ }^{\circledR}$

das umfassende Reinigungsprogramm für Labors aller Bereiche

BORER CHEMIE AG

Zürcher Str. 125

Postfach 352

CH-8952 Schlieren-Zürich

Ruf (01) 7301535

Telex CH 54031 SPONA

- Microliterspritzen ${ }^{\circledR}$

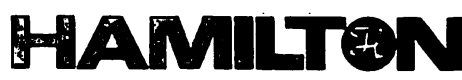

Hamilton Deutschland GmbH

Postfach 110427

Otto-Röhm-Str. 74

D-6100 Darmstadt

Ruf 061 51/85085-86, FS 0419684

- Mikroskope

HERTEL \& REUSS

3500 Kassel, Quellhofstr. 67

Ruf 05 61/8 3006

WILL WETZLAR KG

Optische Werke

Postfach $\mathbf{4 0}$

6331 Nauborn-Wetzlar

Ruf 064 41/2 30.71-4

CARL ZEISS

7082 Oberkochen, Pf. 1369
pH-Meßgeräte + pH-Glaselektroden INGOLD pH-Meßtechnik 6000 Frankfurt 1

Postf. 3308, Ruf 06 11/29 5301

- Photometer

CAŔL ZEISS

7082 Oberkochen, P.f. 1369

- Polarimeter

CARL ZEISS

7082 Oberkochen, Pf. 1369

- Radioaktive Stoffe

AMERSHAM BUCHLER

3300 Braunschweig, Postf. 1120

Ruf 053 07/46 93-97

- Reinigungsmittel

deconex ${ }^{\circledR}$

das umfassende Reinigungspro-

grạmm für Labors aller Bereiche

BORER CHEMIE AG

Zürcher Str. 125

Postfach 352

CH-8952 Schlieren-Zürich

Ruf (01) 7301535

Telex CH 54031 SPONA

\section{- Spektralphotometer}

CARL ZEISS

7082 Oberkochen, Pf. 1369

- Schlauchpumpen

VERDER (DEUTSCHLAND) GMBH 4000 Düsseldorf, Luegallee 108

Ruf 02 11/57 40 79, FS: 08585539

\section{Sterilisierpapiere}

J.C. BINZER

3559 Hatzfeld

Ruf $06467 / 3$ 18, FS: 0482224

- Trockenschränke

HEINICKE INSTRUMENTS

Laborgerätebau $\mathrm{GmbH}$

8223 Trostberg, Pf. 1203

Ruf $08621 / 2085$, FS 563110

\section{- Waschautomaten für}

Laborglas

HEINICKE INSTTRUMENTS

Laborgerätebau $\mathrm{GmbH}$

8223 Trostberg, Pf. 1203

Ruf $08621 / 2085$, FS 563110

\section{Żentrifugen}

BÉR̄THOLD́ HEŔMLE KG

7209 Gosheim; Postfach 1240

Ruf $07426 / 1061$ 
Dic Aufnahme der Chromatogramme erfolgte $5 \mathrm{~min}$ isotherm bei $80^{\circ} \mathrm{C}$, anschließend mit oinem Temperaturprogramm von $4^{\circ} \mathrm{C} / \mathrm{min}$ bis $200^{\circ} \mathrm{C}$. Parameter: Trägergas Wasserstoff, Eingangsdruck $0,38 \mathrm{~kg} / \mathrm{cm}^{2}$. Splitverhältnis 1:20. Detektor: FID, Elektrometereinstellung $1 \times 1 \times 16$. Temperatur des Einspritzblocks: $250^{\circ} \mathrm{C}$.

\section{Massenspektrometrische Untersuchung}

Jeweils $0,4 \mu \mathrm{mol} L$-Hydroxyprolin und allo- $D$-Hydroxyprolin wurden 24 Stunden mit $2 \mathrm{ml} 6 \mathrm{~mol} / 1 \mathrm{HCl}$ bei $110^{\circ} \mathrm{C}$ unter Luftausschluß „hydrolysiert" und wie oben beschreiben derivatisiert.

Die anschließende gaschromatographische Untersuchung zeigte, daß bei der „Hydrolyse" weniger als 1 Prozent Racemisierung zu erwarten ist.

Die Aufnahme der Massenspektren crfolgte in der Kopplung GC/MS nach der Trennung über Chirasil-Val (8) auf einem Massenspektrometer MAT-112 der Firma Varian MAT, Bremen. In der Kopplung GC/MS wurde Helium als Trägergas verwendet.

\section{Parameter für die Messung mittels Elektronenstoß-Ionisation}

Elektronenenergie $80 \mathrm{eV}, 0,7 \mathrm{~mA}$; Temperatur der Ionenquelle $200^{\circ} \mathrm{C}$, Interface $220^{\circ} \mathrm{C}$; SEV-Spannung etwa $1,9 \mathrm{kV}$.

\section{Parameter für die Messung mittels chemischer lonisation}

Reaktionsgas Methan; Elektronenenergie $160 \mathrm{eV}, 0,7 \mathrm{~mA}$; Temperatur der Ionenquelle $200^{\circ} \mathrm{C}$, Interface $220^{\circ} \mathrm{C}$; SEV. Spannung etwa $1,9 \mathrm{kV}$.

\section{Ergebnisse}

Gaschromatographische Trennung von Aminosäuren in biologischem Material

\section{Analyse von Urinproben}

Zur Bestimmung der freien und gebundenen Aminosäuren wurde eine Urinprobe unmittelbar nach der sauren Hydrolyse derivatisiert. Das resultierende Spektrum ist in Abbildung 1 wiedergegeben. Die gefundenen Anteile an $D$-Aminosäuren entstehen während der Hydrolyse der Proben $(9,10)$.

Uberraschenderweise führt die beschriebene Derivatisierung zu den flüchtigen Aminosäurederivaten be- reits zu einem relativ übersich tlichen Spektrum. Durch die fehlende Vorreinigung werden jedoch störende Peaks hervorgerufen, zudem wird die Säule stark belastet. Bei den folgenden Analysen wurde daher in Anlehnung an die Methode von Zumwalt (11) zunächst eine Trennung über einen Kationenaustauscher durchgeführt.

Hochgereinigte Urinproben wurden durch Trennung der Probengemische über einen Kationen- und zusätzlich über einen Anionenaustauscher erhalten (Abb. 2).

\section{Analyse von Plasma}

Die Plasmaproben wurden von Proteinen befreit und ebenfalls über den Kationenaustauscher getrennt (Abb. 3).

\section{Quantitative Hydroxyprolinbestimmungen}

$L$-Hydroxyprolin und allo- $D$-Hydroxyprolin wurden wie im experimentellen Teil beschrieben derivatisiert und einzeln und im Gemisch der gaschromatographischen Enantiomerentrennung unterworfen.

Dabei zeigte sich, daß die verwendete $D$-Standard-Verbindung durch weniger als $0,1 \% L$-Hydroxyprolin verunreinigt war.

In der Kopplung GC/MS wurden zur Absicherung zudem die EI- und CI-Massenspektren aufgenommen, die für beide Verbindungen identisch sind (Abb. 4).

Den Urinproben wurde zur Bestimmung des freien und gebundenen Hydroxyprolins der Standard allo- $D$ Hydroxyprolin nach der sauren Hydrolyse zugesetzt. Die Bestimmungen erfolgten direkt aus dem Urinhydrolysat nach Derivatisierung der Probe, nach der Reinigung des Hydrolysates über den Kationenaustauscher und nach der zusätzlichen Reinigung über den Anionenaustauscher.

Zur Bestimmung des freien Hydroxyprolins in der gleichen Urinprobe wurde die Probe nach Zusatz des Stan-

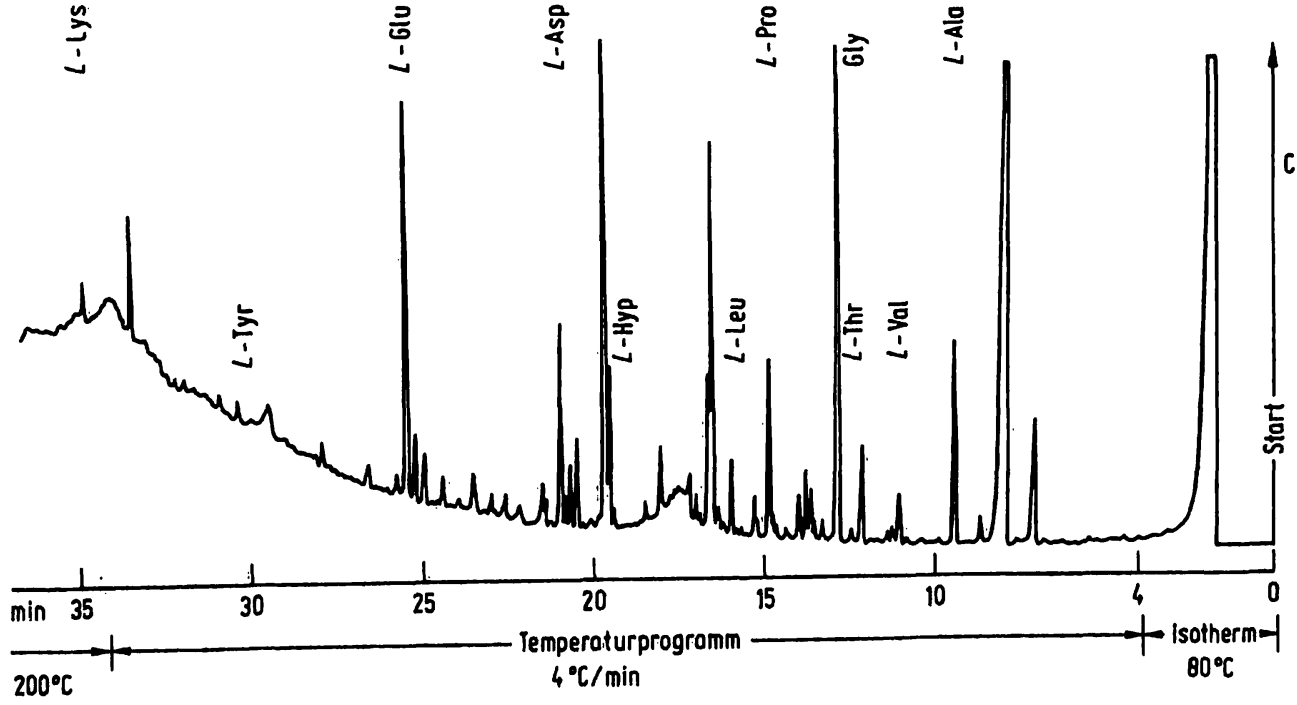

Abb. 1. Chromatogramm nach Hydrolyse und Derivatisicrung der Urinprobc. 


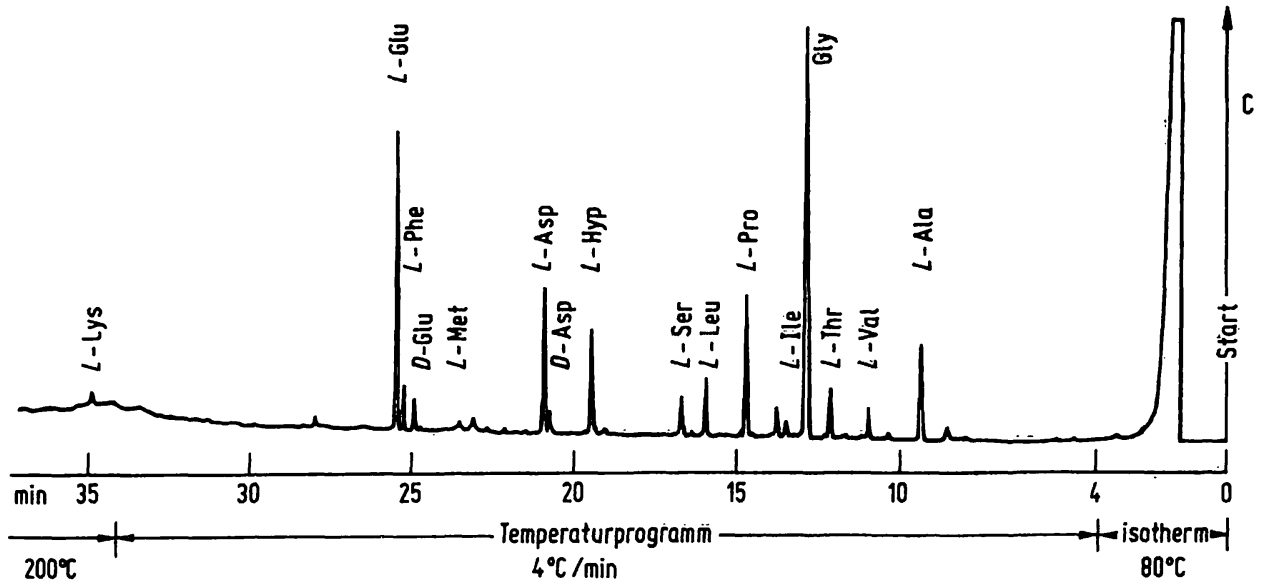

Abb. 2. Chromatogramm nach Hydrolyse, Trennung über Kationen- und Anionenaustauscher und Derivatisierung $4^{\circ} \mathrm{C} / \mathrm{min}$ der Urinprobe.

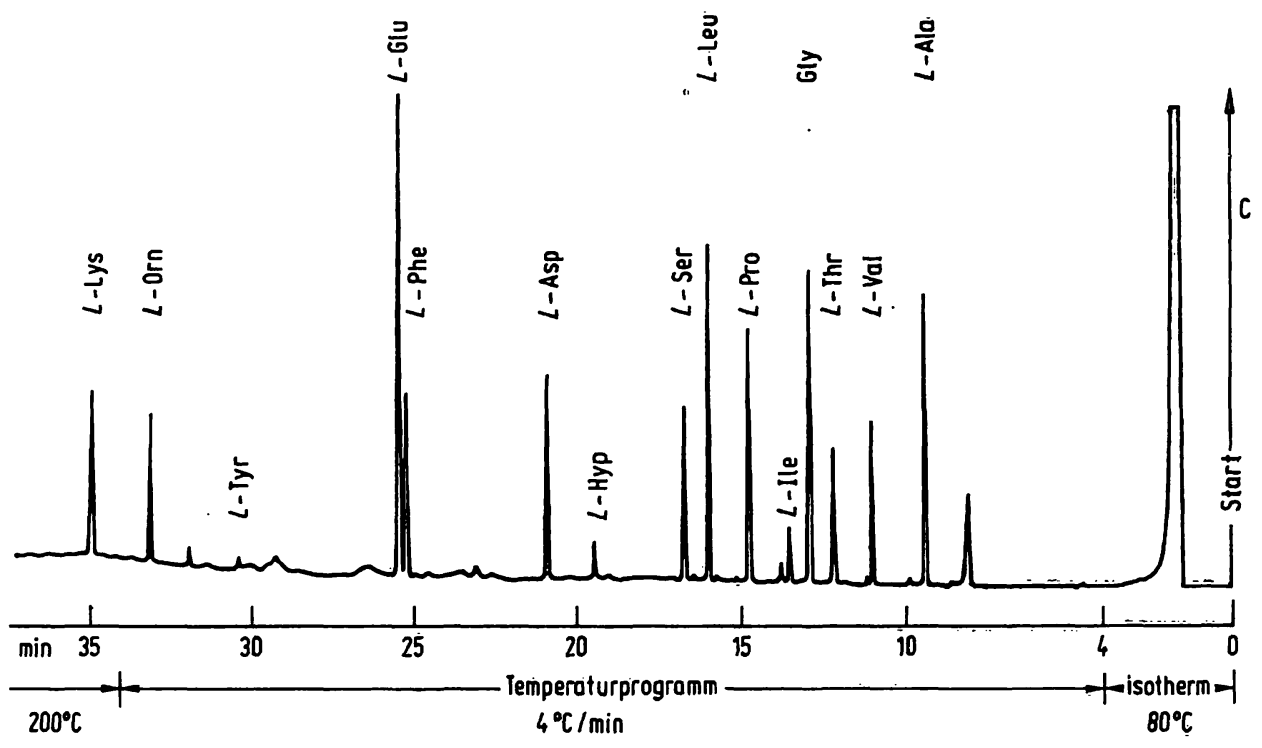

Abb. 3. Chromatogramm nach Pikrinsäurefällung, Trennung über den Kationenaustauscher und Derivatisierung der Plasmaprobe. a) Elektronenstoß - Ionisation

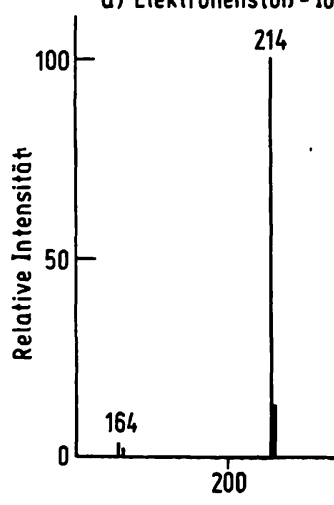

b) Chemische Ionisation

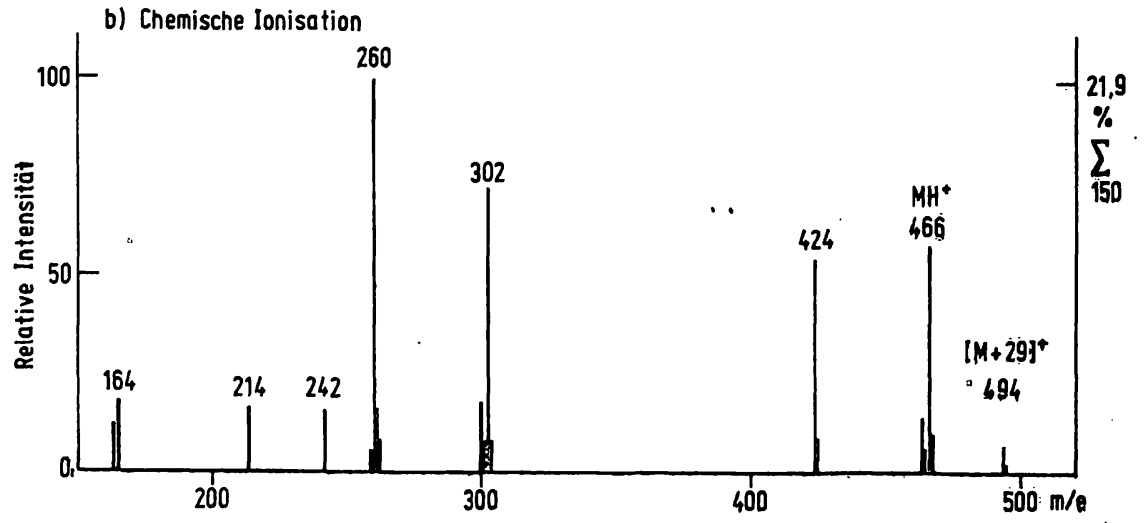

$A b b: 4$. Massenspektren von allo $=\bar{D}-$ und $L \rightarrow$ Hydroxyprolin.

a) Spektrum nach Elektronenstob= Ionisation.

b) Spektrum.nach chemischer Ionisisation. 


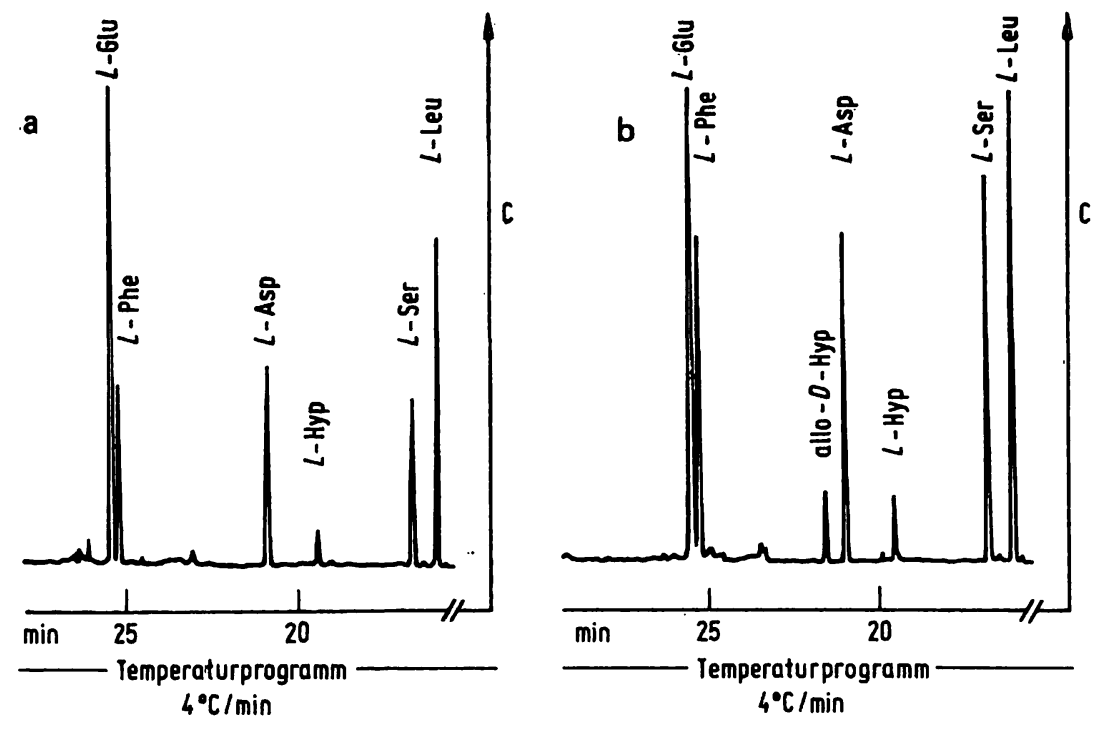
Abb. 5. Ausschnitte aus den Chromato- grammen der Plasmaprobe nach Pikrinsäure- fällung, Trennung über Kationenaustauscher und Derivatisierung.
a) ohne Zusatz.
b) mit Zusatz von $2,5 \mu \mathrm{g}$ allo-D-Hydroxy- prolin.

Tab. 1. Auswertung der quantitativen Hydroxyprolinbestimmungen.

\begin{tabular}{|c|c|c|c|c|}
\hline Probe & $\begin{array}{l}\text { Volu- } \\
\text { men }\end{array}$ & $\begin{array}{l}\text { Reinigungs- } \\
\text { schritt }\end{array}$ & $\begin{array}{l}\text { Zusatz } \\
\text { allo-D- } \\
\text { Hydroxy- } \\
\text { prolin } \\
(\mu \mathrm{g})\end{array}$ & $\begin{array}{l}\text { Gefun- } \\
\text { dene } \\
\text { Menge } \\
L \text {-Hy- } \\
\text { droxy- } \\
\text { prolin } \\
(\mathrm{mg} / \mathrm{l})\end{array}$ \\
\hline $\begin{array}{l}\text { Urin- } \\
\text { hydrolysat }\end{array}$ & 1 & - & 20 & $25,28^{1}$ \\
\hline $\begin{array}{l}\text { Urin- } \\
\text { hydrolysat }\end{array}$ & 1 & $\begin{array}{l}\text { Kationen- } \\
\text { austauscher }\end{array}$ & 20 & $25,24^{1}$ \\
\hline $\begin{array}{l}\text { Urin- } \\
\text { hydrolysat }\end{array}$ & 1 & $\begin{array}{l}\text { Kationen- } \\
\text { austauscher } \\
+ \text { Anionen- } \\
\text { austauscher }\end{array}$ & 20 & $25,10^{1}$ \\
\hline Urin & 1 & $\begin{array}{l}\text { Kationen- } \\
\text { austauscher } \\
+ \text { Anionen- } \\
\text { austauscher }\end{array}$ & 20 & $0,95^{2}$ \\
\hline Plasma & 1 & $\begin{array}{l}\text { Pikrinsäure- } \\
\text { fällung } \\
+ \text { Kationen- } \\
\text { aụstauscher }\end{array}$ & 2,5 & $2,27^{2}$ \\
\hline
\end{tabular}

1 freies und gebundenes Hydroxyprolin

2 freies Hydroxyprolin

dards unter Auslassung des Hydrolyseschrittes über den Kationen- und Anionenaustauscher gereinigt und derivatisiert.

Der Plasmaprobe wurde der Standard noch vor der Pikrinsäurefällung zugefügt. Die teilweise Wiedergabe der Chromatogramme erfolgt in Abbildung 5.

Die Auswertung aller durchgeführten Messungen ist in Tabelle 1 wiedergegeben.

Die Auswertung erfolgte nach dem in der Literatur angegebenen Verfahrẹn (6) über die Peak-Höhen, wobei die während der Hydrolyse auftretende Racemisierung von Probe und Standard korrigiert wird.

Für die Bestimmungen wurden gleiche Mengen einer Urinprobe der jeweiligen Aufarbeitung unterzogen. Die Meßwerte einer Probe waren auf \pm 2 Prozent reproduzierbar, die angeführten Werte sind Mittelwerte aus je 5 Messungen.

Trotz völlig unterschiedlicher Aufarbeitung liegen die gefundenen Werte für freies und gebundenes $L$-Hydroxyprolin in der Urinprobe innerhalb 0,5\% Abweichung vom Mittelwert.

Die Durchführung der Untersuchungen erfolgte an Blutund Urinproben eines Patienten, der sich schwere Verbrennungen zugezogen hatte.

Der Wert von $\sim 2,2 \mathrm{mg} / \mathrm{l}$ für das freie Hydroxyprolin im Plasma ist gegenüber dem Normalwert (14) signifikạnt erhöht.

\section{Diskussion der Ergebnisse}

In den vorausgegangenen Abschnitten wurde die Anwendung der gaschromatographischen Aminosäureanalyse (7) auf die Analyse von biologischem Material beschrieben.

Durch Verwendung des chemisch äquivalenten Standards allo- $D$-Hydrocyprolin, Reinigung der Proben durch chromatographische Verfahren und gaschromatographische Analyse dürfte es sich bei der beschriebenen Methode um eine der genauesten derzeit verfügbaren Hydroxyprolinbestimmungen handeln.

Die bẹnötigten Probemengen können dabei gegenüber bisherigen Bestimmungsmethoden stark reduziert werden.

Der Zeitaufwand für eine komplette Analyse, ausgehend von Urinhydrolysat bzw. Blutplasma, beträgt ca. 2 Stunden fuir die Urinprobe ohne Vorreinigung, 4 Stunden für 
die hochgereinigte Urinprobe und etwa 3 Stunden für die Plasmaprobe. Da die eigentliche gaschromatographische Bestimmung bei Verwendung eines geeigneten Temperaturprogrammes lediglich etwa 20 Minuten dauert, ist ein größerer Probenanfall bei serienweiser Derivatisierung dennoch kurzfristig zu bewältigen.

Der Mehraufwand wird zudem durch den ungleich größeren Informationsgehalt der Spektren kompensiert: wie aus den Abbildungen 1 bis 3 hervorgeht, werden neben Hydroxyprolin gleichzeitig nahezu alle anwesenden $L$-Aminosäuren in einer Messung erfaßt.

Von Baily et al. (15) wurden z. B. neben der ,Leitaminosäure" Hydroxyprolin noch Lysin, Prolin, Alanin und Glycin für die Kollagenbildung bei Meerschweinchen nach der Applikation von Quarzstäuben herangezogen.

\section{Literatur}

1. Franzen, E. (1978), Z. Gesamte Hyg. Ihre Grenzgeb. 24, 12-20.

2. Nagelschmidt, M. \& Struck, H. (1977), Med. Welt 28, 334-335.

3. Stegemann, H. (1958), Hoppe Seyler's Z. Physiol. Chem. $311,41-45$.

4. Haury, H. (1972), Z. Klin. Chem. Klin. Biochem. 10, $25-28$.

5. Mee, J. M. L. (1973), J. Chromatogr. 87, 155-161.

6. Frank, H., Nicholson, G. J. \& Bayer, E., J. Chromatogr., im Druck.

7. Frank, H., Nicholson, G. J. \& Bayer, E. (1977), J. Chromatogr. Sci. 15, 174-176.

8. Frank, H., Nicholson, G. J. \& Bayer, E. (1978), Angew. Chem. 90, 396-398.
Eine quantitative Bestimmung auch dieser Aminosäuren neben Hydroxyprolin in biologischem Material ist nach der hier verwendeten Methode (6) ohne weiteres möglich (16).

Mit der erreichbaren Geñauigkeit sollte es zudem möglich sein, zuverlässige Hydroxýprölin-Normalwerte zu bestimmen, da gerade diesen Punkt betreffen'd die Literaturwerte stark streuen.

\section{Danksagung}

Die Autoren danken Herrn Prof. Dr. E. Bayer, Herrn Dr. H. Frank und Herrn G. Nicholson, Institut für Organische Chemie der Universität Tübingen, für die Überlasșung von Chirasil-Val.
9. Neuberger, A. (1948), Adv. Protein Chem. 4, 339-340.

10. Woiwöde, W., Frank, H., Nicholson, G. J. \& Bayer, E., zur Veröffentlichung eingereicht.

11. Zumwalt, R. W., Roach, D. \& Gehrke, C. W. (1970), J. Chromatogr. 53, 171-194.

12. Schulte, E. (1976), Chromatographia 9, 315-320.

13. Chirasil-Val ist erhältlich von Applied Science Laboratories, P. O. Box 440, College Station, Pennsylvania 16801 (USA).

14. Documenta Geigy, Wissenschaftliche Tabellen 7. Auflage (1968), Herausgeber: J. R. Geigy A. G., Pharma, Basel.

15. Baily, P., Kilroe-Smith, T. A. \& Harington, J. A. (1964), Arch. Environ. Health 8, 547-554.

16. Woiwọde, W., Weichardt, H., Nicholson, G. J. \& Bayer, E., unveröffentlichte Ergebnisse.

Prof. Dr. med. H. Weichardt Institut für Arbeits- und Sozialmedizin der Universität Tübingen Wilhelmstraße 27 D-7400 Tübingen 